\title{
Performance analysis of prioritized handoff schemes in wireless systems
}

\author{
Uduak Idio Akpan ${ }^{1, ~ *, ~ C o n s t a n c e ~ K a l u ~}{ }^{1}$, Aniebiet Kingsley Inyang ${ }^{2}$ \\ ${ }^{1}$ Department of Electrical /Electronics and Computer Engineering, University of Uyo, Akwa Ibom State, Nigeria \\ ${ }^{2}$ Department of Electrical and Electronics Engineering, Federal University of Technology, Owerri, Imo State, Nigeria
}

Email address:

idioh1@yahoo.com (U. I. Akpan), const4@yahoo.com (C. Kalu), Aniebiet.kingsley@yahoo.com (A. K. Inyang)

\section{To cite this article:}

Uduak Idio Akpan, Constance Kalu, Aniebiet Kingsley Inyang. Performance Analysis of Prioritized Handoff Schemes in Wireless Systems. Communications. Vol. 2, No. 1, 2014, pp. 1-6. doi: 10.11648/j.com.20140201.11

\begin{abstract}
The rapid increase in the use of mobile devices demands the need to meet Quality of service requirements of the users. These requirements however, (application demand and allocation) often lead to network congestion and call drops. To deal with this issues (call drop and congestion), many prioritized handoff schemes have been proposed by many researchers. In our previous paper entitled "Development of an Improved Scheme for Minimizing Handoff Failure due to Poor Signal Quality", we proposed a prioritized handoff scheme which integrates the direction of movement of the mobile device to the $\mathrm{M}+\mathrm{G}$ scheme in making handoff decision. In this paper, we make comparative analysis of that scheme by comparing its performance with other existing schemes. The MatLab software was used for the simulation.
\end{abstract}

Keywords: Handoff Failure, Mobility Factor, Mobile Networks, Quality of Service, Congestion

\section{Introduction}

In the few years, the wireless network has experienced tremendous advancement globally. It is quite likely that there will be even more advancement in years to come. This advancement is not just in the increase in the number of users $(1,2,3)$, but also on the demands on other applications. This results in ever greater resource requirements. The design of such a network, which is based on a cellular architecture $(4,5,6)$, will give room for adequate use of the available frequency spectrum. It is usually advised that the wireless channel be kept from reaching the congestion point, since it will cause an overall channel quality to degrade and loss rates to rise. Also, the demand for wireless connectivity has increased in mobile wireless cellular networks and service providers need to accommodate more number of users within the limited available bandwidth. Allocated bandwidth has to be utilized properly. Wastage of bandwidth leads to reduction in revenue generation for service provider. Bandwidth allocation, efficient resource utilization and management in the cell are the most concerned issues and many researchers proposed solutions in this regard. During communication a mobile device always remains within the range of at least one base station (BS). Due to the limited spectrum, cellular systems distribute smaller cells in order to achieve high system capacity. Some common problems associated with channel congestion are call block and call drops. The latter leads to forced termination call. The forced termination of an ongoing call is considered less desirable than blocking the initial access of a new call. For a mobile station to communicate with another user or a base station, it must first obtain a channel from one of the base stations that it is talking to. If there is available channel, it is granted to the user otherwise the new call will be rejected. The channel is released either when the user completes the call or moves to another cell before the call is completed. The process whereby a mobile user moves from one cell to another while a call is in progress is known as handoff. While performing handoff mechanism, the mobile unit requires that the base station in the cell that it moves into will allocate it a channel (4). Handoff mechanism is a key element in the provision of guaranteed quality of service in wireless networks. Two important measures in assessing the QoS performance of a mobile system are the new call blocking probability and call dropping probability. The latter is largely as the result of handoffs failures. Most times, the poor QoS experienced in wireless network systems is attributed largely to handoff defects and has caused many mobile users to subscribe to more than one service provider in order to maintain seamless connection. Issues like call admission, connection quality, handoff 
success and mobility management determine the users' satisfaction $(8,9)$.

\section{Review of Related Literature}

Several handoff strategies have been proposed in the literatures over the years. A new handoff technique that combined the Mobile Assisted HandOff (MAHO) and Guard Channels (GC) techniques was proposed in (20). This technique demands that the mobile terminal (MT) reports back not only the Received Signal Strength Indicator (RSSI) and the Bit Error Rate (BER) but the number of free channels that are available for the handoff traffic as well. This is required to ensure that the handed off call meets both the acceptable signal quality standard and the free available channel demands. Furthermore, analytical model was used to obtain the desired performances measures in terms of call blocking and dropping probabilities in the analysis. The analysis showed that ignoring the effects of poor signal quality handoff calls can results in deterioration in performance. To handle the poor signal quality handoff, their work describes two new handoff techniques; the $\mathrm{M}+\mathrm{G}$ (MAHO \& GC) approach and the rehandoff. They latter maintains that, it is better to rehandoff poor quality handoff calls to some other Base Station System (BSS) instead of dropping such calls. (7) presents a method for improving the quality of service (QoS) in multimedia wireless systems based on prioritization of handover requests. A strategy called signal strength for multimedia communications (SSMC) is proposed. In this strategy, the authors calculated a handoff priority for every multimedia service using three values: the static priority value, the degradation rate of the received signal strength ( $\Lambda \mathrm{RSS})$, and the RSS level itself. Then, each handoff request is queued and handled according to its priority value. They presented the detailed algorithm and analyzed its performance on a 25-cell network. The performance of their scheme was compared with other methods by simulations. The results indicated their method can effectively reduce the handoff call dropping probability compared to nonpriority schemes.

(10) Proposed an efficient dynamic fair resource allocation scheme for supporting multimedia traffic in the uplink of wideband CDMA cellular networks with QOS satisfaction and showed that the proposed scheme enhances radio resource utilization and guarantee statistical QOS under different fairness bound requirements. (11) Proposed two handoff schemes without and with preemptive priority procedures in integrated wireless networks and observed that the forced termination probability of voice handoff request calls can be reduced by increasing the number of reserved channels and by employing a preemptive priority handoff scheme. (12) gave the priority to handover calls over new call attempts and blocked handover call are placed in a finite storage queue and evaluated the total handover forced termination probability.[13] Proposed four schemes: SFTT (Single-Queue, FIFO, Timeout, Average Timeout) scheme,
SPTT (Single-Queue, Priority, Timeout, Average Timeout) scheme, DFTS (Dual-Queues, FIFO, Timeout, Statistical TDM) scheme, DPTS (Dual Queues, Priority, Timeout, Statistical TDM) scheme to reduce call completion rates and compared these schemes with NPS and FIFS. Handoff schemes with non preemptive and preemptive channel borrowing to improve resource utilization while keeping a good isolation among different services is proposed in (14). Three different channel allocations schemes NPC, FSC and FRC and channel allocation models to analyze the channel allocation process for wireless networks discussed and shows that the usage of reserved channel can improve the GOS (Grade of Service) of base station greatly in (15). Nasif and his coauthors (19), gave an overview about issues related to handoff initiation and decision. In that paper, different approaches were proposed and applied in order to achieve better handoff service. Forced termination probability and call blocking probability are employed as principal parameters used to evaluate handoff techniques. Mechanisms such as guard channels and queuing handoff calls decrease the forced termination probability while increasing the call blocking probability. Different types of handoff techniques: the soft and hard handoff techniques are also discussed in their work.

\section{Implementation and Simulation}

In (16) we developed an Improved Scheme for Minimizing Handoff Failure Due To Poor Signal Quality. We adopted the $\mathrm{M} / \mathrm{M} / \mathrm{S} / \mathrm{S}$ approach to model the system. We also analysed the performance of this new scheme in terms of new call blocking and handoff failure probabilities. In this paper, the performance of the proposed scheme will be compared with some existing schemes. We built Simulation environments in MatLab for the comparative evaluations of performance of the proposed schemes and with other schemes notably $\mathrm{M}+\mathrm{G}, \mathrm{GC}, \mathrm{MAHO}$ and $\mathrm{M}+\mathrm{Re}$.

\subsection{Brief Model Description}

We modeled the system using the analytical modeling approach (16). The system model is shown in figure 1 . Two traffic request types were considered in the analysis, namely; the new calls and handoff call requests. The following two assumptions were adopted in the system model:

(i) The new call and Handoff rates in the cell are assumed to form a Poisson process with mean values of $\lambda_{\mathrm{N}}$ and $\lambda_{\mathrm{H}}$ respectively.

(ii) The new call and handoff completion time are exponentially distributed with mean rates of $\mu_{\mathrm{N}}$ and $\mu_{\mathrm{H}}$ respectively.

To maximize the priority given to handoff calls, the mobility concept that considers the direction and speed of the MT was used in this scheme alongside the $\mathrm{M}+\mathrm{G}$. This is a concept where a poor signal handoff request is accepted with probability of $\alpha$ if the MT is approaching the BS. The notion behind this is that the signal is assumed to improve 
as the MT gets closer to the BS. We denoted this factor as $\alpha$ and it lies between zero (0) and one (1). The state transition diagram for the model is as depicted in Fig. 2.

Utilizing the queuing theory approach, we developed a mathematical model for the scheme. The handoff failure probability $\mathrm{P}_{\mathrm{HF}}$ was given as

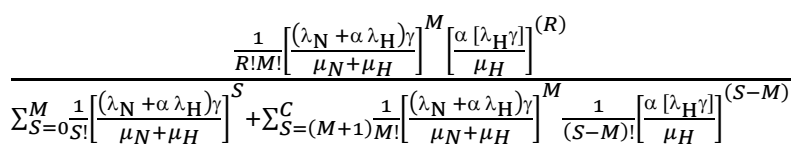

Where, $\lambda_{\mathrm{N}}$ and $\lambda_{\mathrm{H}}$ are the arrival rates of new calls and handoff calls respectively. $\mu_{N}$ and $\mu_{H}$ are the service rates for new calls and handoff calls respectively. $\alpha$ and $\gamma$ are the mobility and signal strength factors respectively. $\mathrm{R}$ and $M$ represent the reserved and shared channels respectively. $\mathrm{C}$ is the number of channels. $\mathrm{S}$ represents the states which is usually

$$
\mathrm{S}=\mathrm{C}+1
$$

Also, for better understanding, we presented a flow chart of the system algorithm implementation. This is shown in figure 3 .

The system parameters used for the simulation is as shown in table1.

The interface (GUI) for the simulation is shown in figure 4.

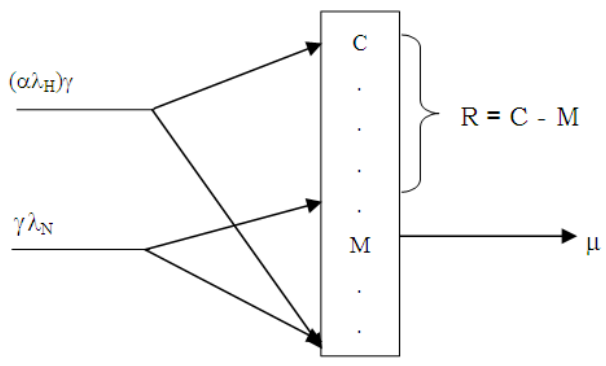

Figure 1. System Model

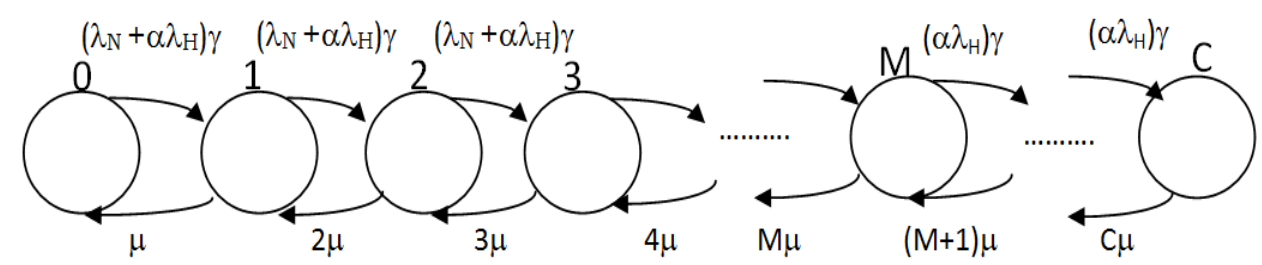

Figure 2. State Transition Diagram

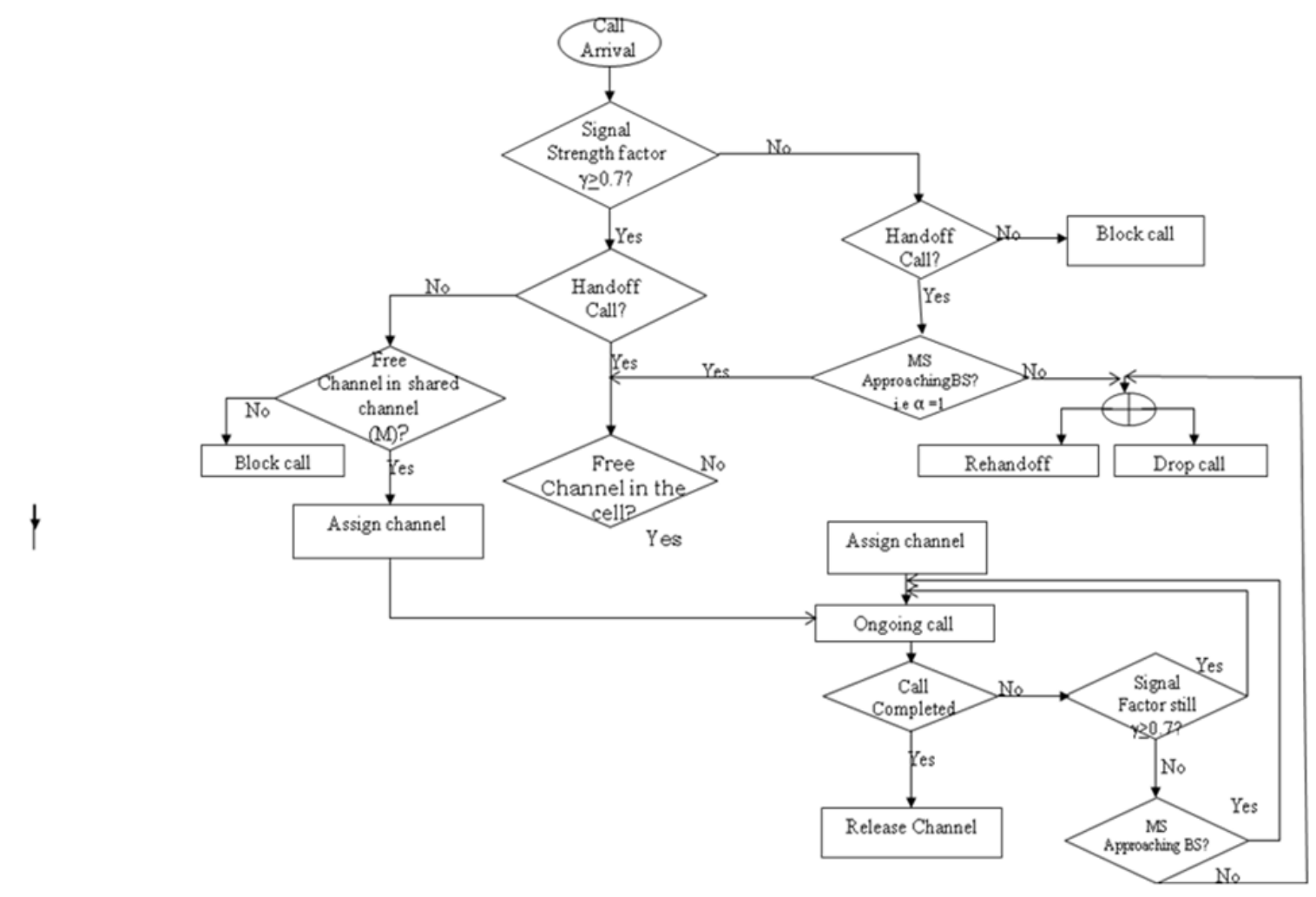

Figure 3. Flow Chart for the Model 


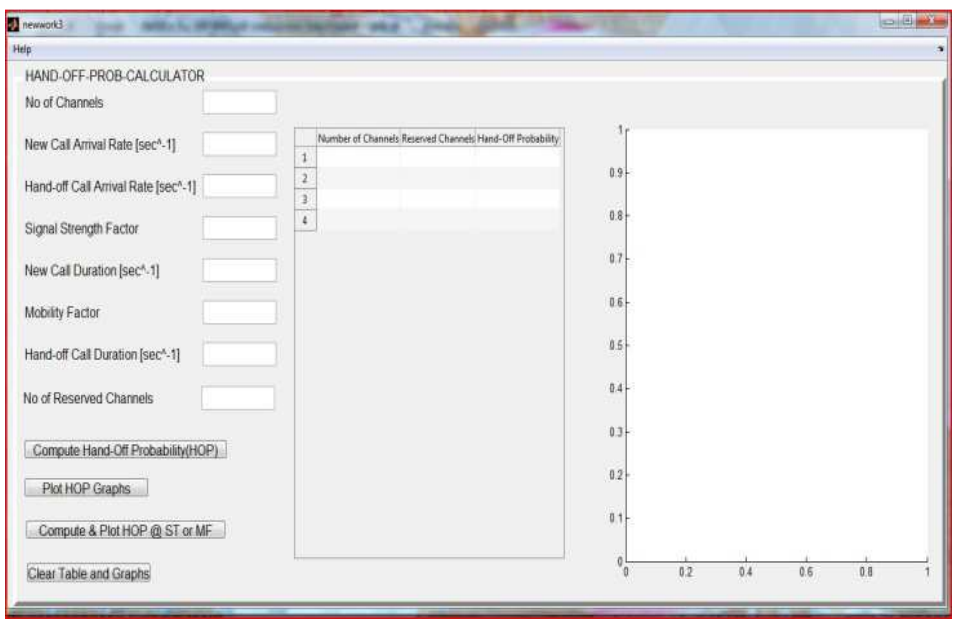

Figure 4. Simulation GUI

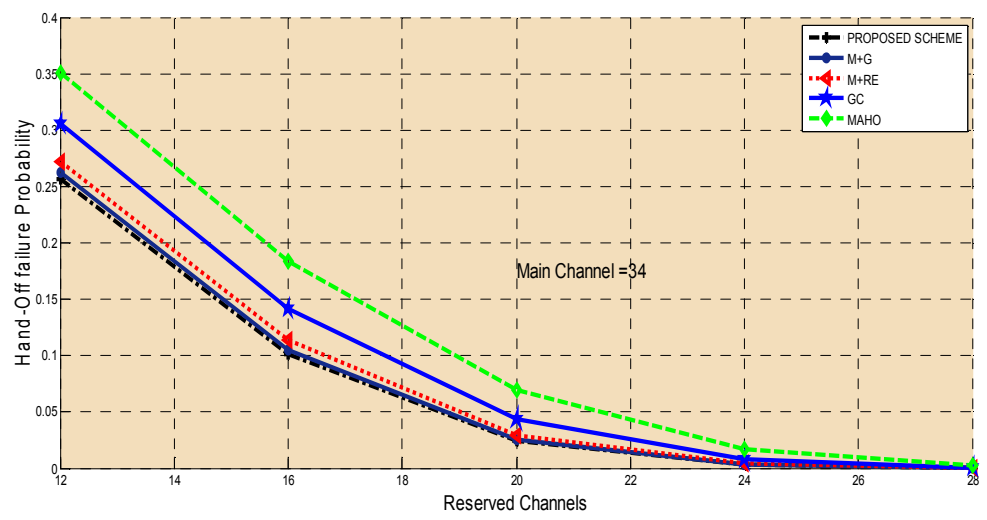

Figure 5. Handoff Failure Probability Vs Reserved Channel Size

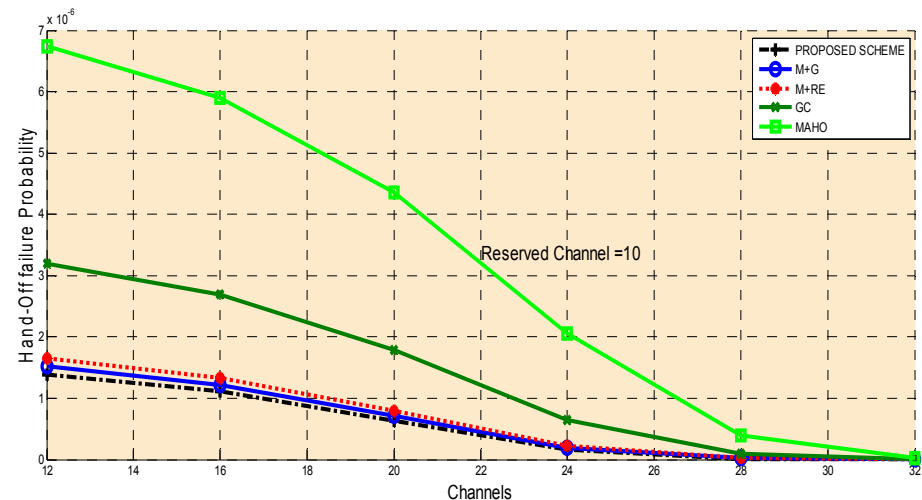

Figure 6. Handoff Failure Probability Vs Total Channel Size

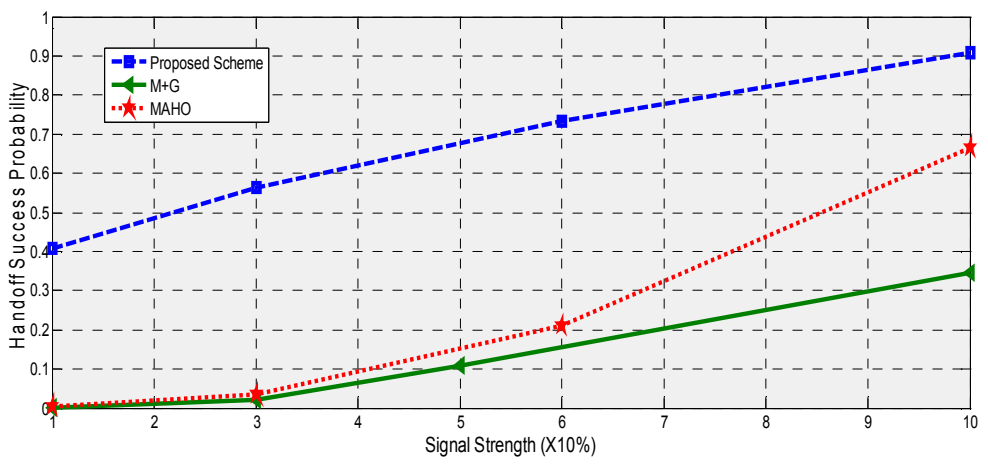

Figure 7. Handoff Failure Probability Vs Signal Strength 


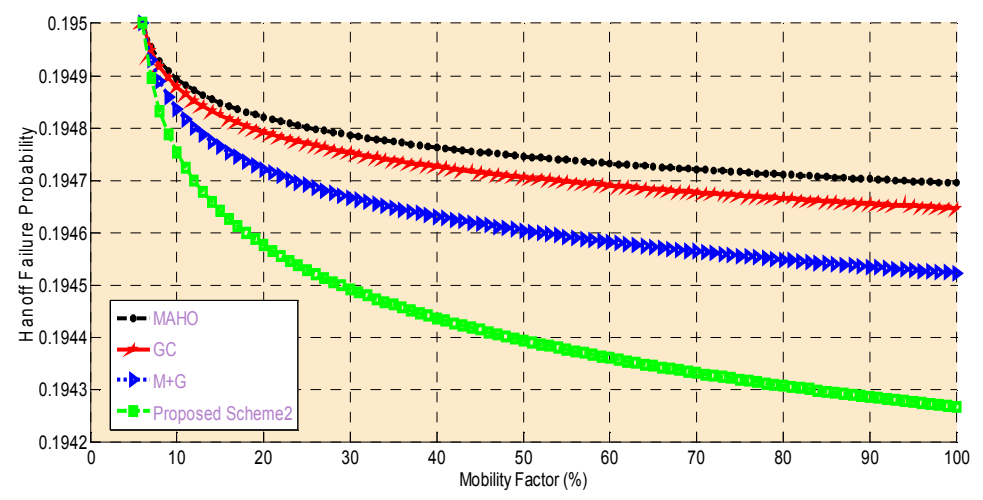

Figure 8. Handoff Failure Probability Vs Mobility Factor

Table 1. System Parameters

\begin{tabular}{ll}
\hline Parameter & Value \\
\hline New call arrival $\left(\lambda_{\mathrm{N}}\right)$ & $1.5 / \mathrm{s}$ \\
Handoff arrival $\left(\lambda_{\mathrm{H}}\right)$ & $1.5 / \mathrm{s}$ \\
Signal strength factor $(\gamma)$ & Varied from $0.1-0.9$ \\
Number of Channels & Varied from $1-32$ \\
Number of Reserved (Guard) channels & Varied from $6-28$ \\
Mobility Factor $(\alpha)$ & Varied from $0.1-0.9$ \\
New call duration $\left(1 / \mu_{\mathrm{N}}\right)$ & $70(\mathrm{~s})$ \\
Handoff call duration $\left(1 / \mu_{\mathrm{H}}\right)$ & $70(\mathrm{~s})$ \\
\hline
\end{tabular}

\section{Comparative Analysis}

In this section, the performance of the proposed scheme is compared with some existing handoff schemes viz; $\mathrm{M}+\mathrm{G}$, $\mathrm{M}+\mathrm{Re}, \mathrm{GC}$ and MAHO. The display in figures 5 and 6 show the comparison with respect to the number of reserved channels and the total channels respectively. To show the improvement provided by the new handoff scheme, the relative performance of the four different handoff schemes are compared.

1) GC refers to the simple Guard Channel approach any poor-signal-quality handoff call received by a BSS cannot be sustained by the BSS and is simply dropped.

2) "MAHO" has no guard channels. This scheme makes decision only on received signal strength.

3) The "G + ReHo" scheme in which poor-quality handoff calls are re-handed off.

4) The "M+G" scheme that utilizes the handoff protocol combining the MAHO and GC, which leads to $\gamma \rightarrow 1$.

5) The proposed channel which utilizes the features in $\mathrm{M}+\mathrm{G}$ alongside the consideration of the direction of the mobile terminal in making handoff decisions, which leads to $\alpha \rightarrow 1$ and $\gamma \rightarrow 1$.

It can be seen from Figures 5 and 6 that the handoff failure probability of the proposed scheme is lower than that of the other schemes. The plots show that the proposed scheme out performs other schemes under study. This is because the proposed scheme takes into consideration the channel availability, signal quality and the direction of movement of the MS in making handoff decision. This ensures that fewer handoff calls are dropped. In figure 7, the effect of signal quality on handoff success is depicted. The figure shows that as the signal quality improves, the handoff success probability increases. Here also, the proposed scheme has a better performance than the other schemes. The proposed scheme is based on the idea that if the mobile terminal is approaching the base station, the handoff request signal will improve as it nears the base station. Figure 8 demonstrates the effect of the mobility factor $(\alpha)$ on the handoff failure probability for both the GC scheme and the new handoff scheme. Also, it can be observed that the handoff failure probability decreases as the value of mobility factor increases rapidly for the proposed scheme as compared to other schemes. This is because, the proposed scheme through mobile assistance and updates ensures that $\alpha$ is always one (1) before the handoff request can be accepted. The value of one (1) for the factor depicts that the MS is approaching the BS thereby causing improvement in the signal quality. It can be observed that the handoff probability for all schemes converges to the same point as the mobility factor tends to zero $(0)$.

\section{Conclusion}

The comparative performance of various handoff schemes in terms of handoff failure and success probabilities was carried out in this paper. The performance of these schemes were evaluated and compared with our previously proposed handoff scheme in [16] using MatLab. It has been demonstrated through simulations that the proposed scheme performs better than the other schemes. The proposed scheme is based on the idea that if the mobile terminal is approaching the base station, the poor signal handoff request will improve as the mobile terminal approaches the base station. In essence, this scheme ensures that $\alpha$ is always one before accepting the handoff request.

\section{References}

[1] D.L Schilling, "Wireless Communications going into the 21st Century," IEEE Transactions on Vehicular Technology, vol. 43, no 3, pp. 645- 651, 1994.

[2] J. E Padgett, C.G Gunther and T. Hattori, "Overview of Wireless Personal Communications," IEEE Communications Magazine, pp. 28-41, 1995. 
[3] D. C Cox, "Wireless Personal Communications: What is it?," IEEE Personal Communications, vol.2, no.2, pp.20-35, 1995.

[4] D. J. Goodman, "Cellular Packet Communications", IEEE Transactions on Communications," vol. 38, pp. 1272-1280, 1990.

[5] [5] L. Cidon and M. Sidi, "A Multi-Station Packet-Radio Network," Performance Evaluation, vol. 8, no.1, pp.65-72, 1988.

[6] W. Lee, Mobile Cellular Telecommunications Systems, New York: McGraw-Hill, 1989.

[7] R. Chang and S. Leu, "Handoff Ordering Using Signal Strength for Multimedia Communications in Wireless Networks," IEEE Transactions on Wireless Communications, vol. 3, no. 5, September, 2004.

[8] A. Felipe, H. Genaro and R. Andrés, "Call-Level performance sensitivity in cellular networks," Electrical Engineering Department, CINVESTAV-IPN Electronics Department, UAM-A Mexico, 2010.

[9] Y. Fang and Y. Zhang, "Call admission control schemes and performance analysis in wireless mobile networks," IEEE Transactions on Vehicular Technology, vol. 51, no. 2, March, 2002.

[10] W. Li, H. Chen and D.P. Agrawal, "Performance Analysis of Handoff Schemes with Preemptive and Non-preemptive Channel Borrowing in Integrated Wireless Cellular Networks," IEEE Transactions on Wireless Communications, no. 4, pp. 1222-1233, 2005.

[11] S. Louvros, J. Pylarinos ans S. Kotsopoulos, "Handoff Multiple Queue Model in Microcellular Networks," Computer Communications, no. 30, pp. 396-403, 2007.

[12] A. Sgora and D.D. Vergados, "Handoff Prioritization and Decision schemes in Wireless Cellular Networks: A Survey, IEEE Communications Surveys and Tutorials," no.11, pp.57-77, 2009.

[13] K. Venkatachalam and P. Balasubramanie, "A Hybrid Resource Allocation Strategy with Queuing in Wireless
Mobile Communication Networks," Computer and Information Science, no.2, pp. 3-14, 2009.

[14] [L. Xu, X. Shen and J. H. Mark, "Fair Resource Allocation with Guaranteed Statistical QOS for Multimedia Traffic in Wideband CDMA Cellular Network", IEEE Transactions on Mobile Computing," no.4, pp.166-177, 2005.

[15] Q.-A. Zeng and D. P. Agrawal, "Modeling and Efficient Handling of Handoffs in Integrated Wireless Mobile Networks," IEEE Transactions on Vehicular Technology, no.51, pp. 1469-1478, 2002.

[16] U. I. Akpan, C. Kalu, S. Ozuomba and A. Obot, (2013), "Development of an Improved Scheme for Minimizing Handoff Failure due to Poor Signal Quality," International Journal of Engineering Research and Technology vol 2, no.10, pp. 2764-2771, 2013.

[17] Y. Fang, "Performance evaluation of wireless cellular networks under more realistic assumptions," Wireless Communications and Mobile Computing Wirel. Commun. Mob. Comput.; vol 5, pp.867-885 Published online in Wiley Interscience (www.Interscience.Wiley.Com). DOI: 10.1002/Wcm.352, 2005.

[18] S. Dharmarajaa, K. S. Trivedib, and D. Logothetisc, "Performance modeling of wireless networks with generally distributed handoff interarrival times," Computer Communications vol. 26, pp.1747-1755, 2003.

[19] E. Nasif, S. Tara, K. Sibel and F. Kemal, "An overview of handoff techniques in cellular networks," International Journal of Information and Communication Engineering vol. 2 pp.6, 2006.

[20] B. B. Madan, S. Dharmaraja and K. S. Trivedi, "Combined guard channel and mobile-assisted handoff for cellular network,"; IEEE Transactions on Vehicular Technology, vol. 57 , no. $1,2008$.

[21] V. Goswami and P. K. Swain, "Analytical modeling for handling poor signal quality calls in cellular network," International Journal of Networks and Communications 2012, vol. 2, no.4, pp.47-54, 2012. 